\title{
ISOLATION OF BIOACTIVE COMPOUNDS FROM DICRANACEAE MOSSES
}

\author{
Junairiah Junairiah $^{1 *}$, Tri Nurhariyati ${ }^{1}$, Ni'matuzahroh ${ }^{1}$, Lilis Sulistyorini ${ }^{2}$ \\ ${ }^{1}$ Department of Biology, Faculty of Science and Technology, Airlangga University, Surabaya \\ ${ }^{2}$ Faculty of Public Health, Airlangga University, Surabaya \\ email: alip.jun1@gmail.com
}

Received 3 July 2016

Accepted 30 November 2016

\begin{abstract}
Dicranoloma reflexum and Dicranella coarctata are mosses from Dicranaceae family. This study was purposed to identify bioactive compounds contained from both species. Dicranoloma reflexum and Dicranella coarctata collected form Cangar forest, Batu, East Java. Mosses was rinsed, dried and crushed into powder. Extraction was performed using maceration method with n-hexane, acetic acid, and methanol solvent. Compounds obtained then identified using Gass Chromatography Mass Spectra. Result showed that nhexane, acetic ethyl, and methanol extract of Dicranoloma reflexum contained 61, 16, and 58 compounds respectively. Main component of each extract was 1-octadecene, phenol, and 9-octadecanoic acid. N-hexane, acetic ethyl, and methanol extract of Dicranella coarctata contained 5, 38, and 23 compounds respectively. Main component of each extract was thiosulphuric acid, E-15 heptedecenal, and n-hexadecanoic acid.
\end{abstract}

Keywords : Dicranaceae, bioactive compounds

\section{Introduction}

Bryophyte or mosses is source for various secondary metabolites (Asakawa, 2008). Mosses from Bryophyte is rich on secondary metabolites, such as terpenoid, flavonoid, and bibenzyles, also other compounds like fatty acid (Asakawa, 2007). With such secondary metabolites contents, many species of Bryophyta possess various biological activities, such as anti-bacteria, anti-fungal, anti-oxidant, and anti-inflammatory properties (Saxena dan Harinder, 2004).

For example Indian mosses Bryum argenteum had anti-pyretic, anti-rhynitic, and anti-bacterial properties. Himalayan Fissidens laxitextus has been known as an anti-diuretic. Funaria hygrometrica from India was also used as medicine for skin disease. Entodon myurus was found to be potential as anti-bacterial agent towards
Enterobacter aerogenes and Klebsiella pneumoniae (Alam et al., 2015).

Dicranuloma reflexum and Dicranella coarctata are mosses species from Bryopsida (Musci) class, Dicranales order, and Dicranaceae family. Both species has different habitat. Dicranuloma reflexum is arboreal moss, while Dicranella coarctata is terrestrial moss. Up until now, bioactive compounds from both species has yet to be studied. This study was aimed to isolate and identify bioactive compounds from Dicranuloma reflexum and Dicranella coarctata. This study was expected to give new lights on bioactive compounds contained on both moss species as basic information which then used for evaluating biological activities. 


\section{Material and method}

Main ingredient used on this study was moss gametophyte of Dicranoloma reflexum and Dicranella coarctata. Both species was collected from Cangar forest, Batu, Malang, East Java.

\section{Extraction}

Moss gametophyte Dicranoloma reflexum and Dicranella coarctata was rinsed, dried, cut into small pieces, and weighted. Total weigh of Dicranoloma reflexum collected was $17.1 \mathrm{~g}$, while Dicranella coarctata weighted $4.5 \mathrm{~g}$. Moss was divided into three parts, each weighted $5.7 \mathrm{~g}$ and $1.5 \mathrm{~g}$. Each part extracted using n-hexane, acetic ethyl, and methanol. Solvent volume used for extraction of Dicranoloma reflexum and Dicranella coarctata were $200 \mathrm{ml}$ and $100 \mathrm{ml}$ respectively. Extraction was performed using maceration method. Simplicia soaking was conducted for 4 days and repeated for 4 times. Each extract of Dicranoloma reflexum and Dicranella coarctata was dried until all solvent had evaporated perfectly.

\section{Bioactive Compound Identification}

Each extract obtained was analyzed using Gass Chromatography Mass Spectra.

\section{Results and discussion}

Dicranoloma reflexum and Dicranella coarctata were mosses lived on arboreal and terrestrial habitat respectively (Fig 1 and 2).

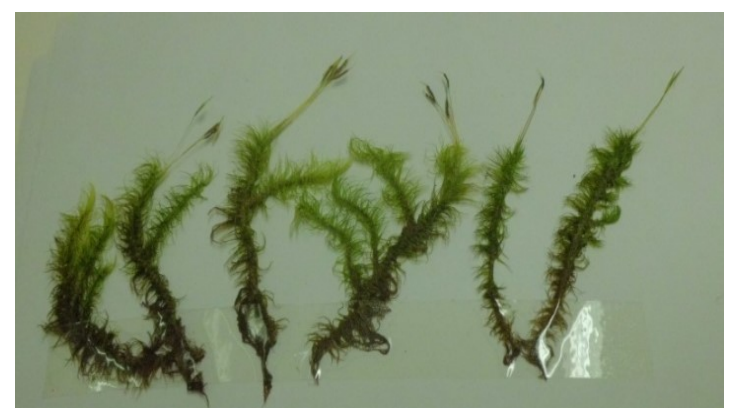

Figure 1. Morphology of Dicranoloma reflexum moss.

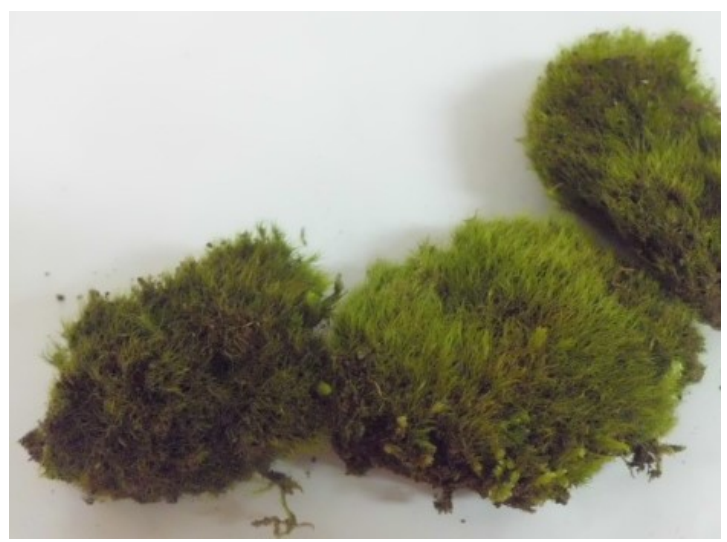

Figure 2. Morphology of Dicranella coarctata moss.

Extract obtained from Dicranoloma reflexum and Dicranella coarctata were presented on Table 1.

Table 1. Extraction of Dicranoloma reflexum and Dicranella coarctata using various solvent.

\begin{tabular}{cllclll}
\hline No & Moss species & $\begin{array}{c}\text { Simplicia } \\
\text { weigh }(\mathrm{g})\end{array}$ & $\begin{array}{c}\text { Solvent } \\
\text { volume } \\
(\mathrm{ml})\end{array}$ & $\begin{array}{c}\text { N-hexane } \\
\text { extract weigh }(\mathrm{g})\end{array}$ & $\begin{array}{c}\text { Acetic ethyl } \\
\text { extract weigh } \\
(\mathrm{g})\end{array}$ & $\begin{array}{c}\text { Methanol extract } \\
\text { weigh }(\mathrm{g})\end{array}$ \\
\hline 1 & $\begin{array}{l}\text { Dicanoloma } \\
\text { reflexum } \\
\text { Dicranella } \\
\text { coarctata }\end{array}$ & 5.7 & 200 & 0.1531 & 0.0851 & 0.7110 \\
\hline
\end{tabular}

GCMS Analysis of Dicranoloma reflexum and Dicranella coarctata Extract 
Based on GC-MS analysis, chromatogram profile of $n$-hexane, acetic ethyl, and methanol of Dicranoloma reflexum and Dicranella coarctata were presented on Figure 2, 3, 4, 5, and 6.

Result of identification on bioactive compounds from n-hexane, acetic ethyl, and methanol extract were presented on table 2, 3, 4, 5, 6, dan 7. N-hexane, acetic ethyl, and methanol extract was found to contain 61, 16, and 58 compounds respectively (Table 2, 3, and 4). Nhexane, acetic ethyl, and methanol extract of Dicranella coarctata contained respectively 5, 38, and 23 compounds (Table 5, 6, and 7).

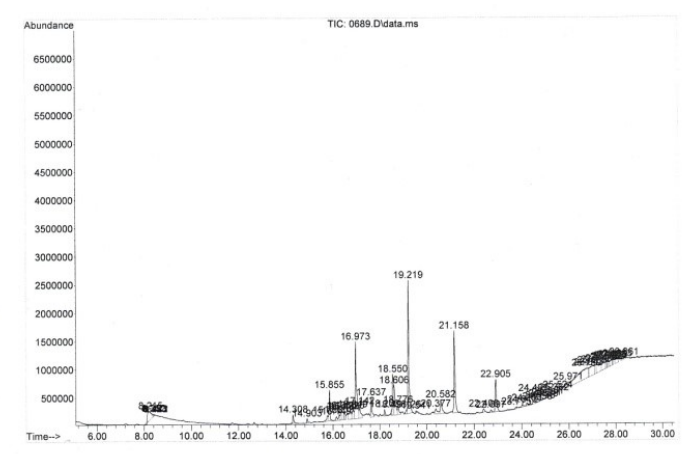

Figure 2. Chromatogram profile of nhexane extract from Dicranoloma reflexum

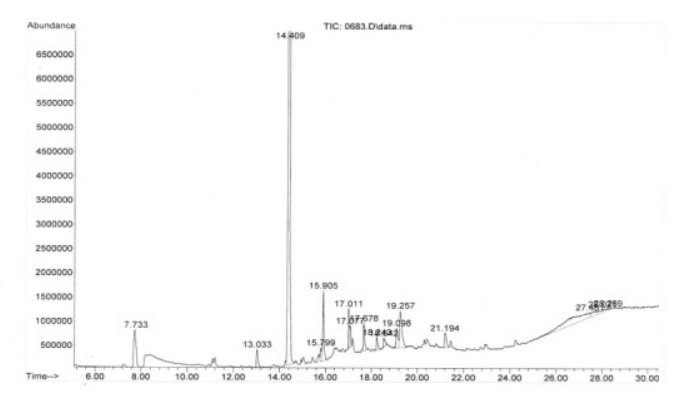

Figure 3. Chromatogram profile of acetic ethyl extract from Dicranoloma reflexum

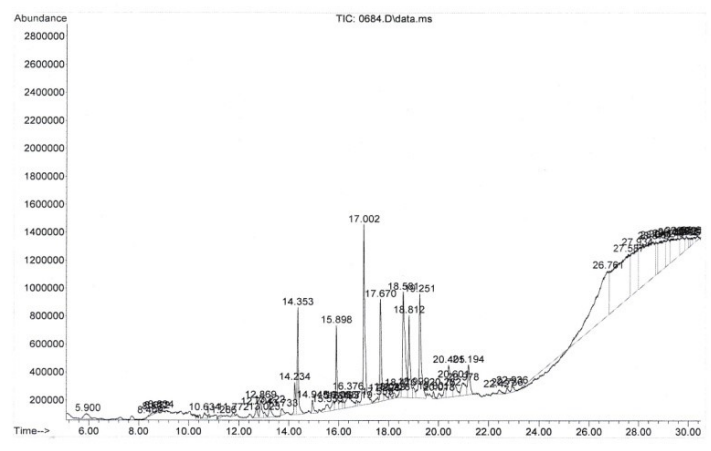

Figure 4. Chromatogram profile of methanol extract from Dicranoloma reflexum

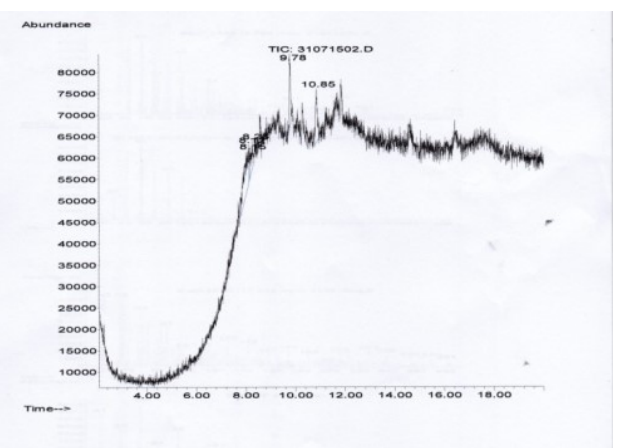

Figure 5. Chromatogram profile of nhexane extract from Dicranella coarctata

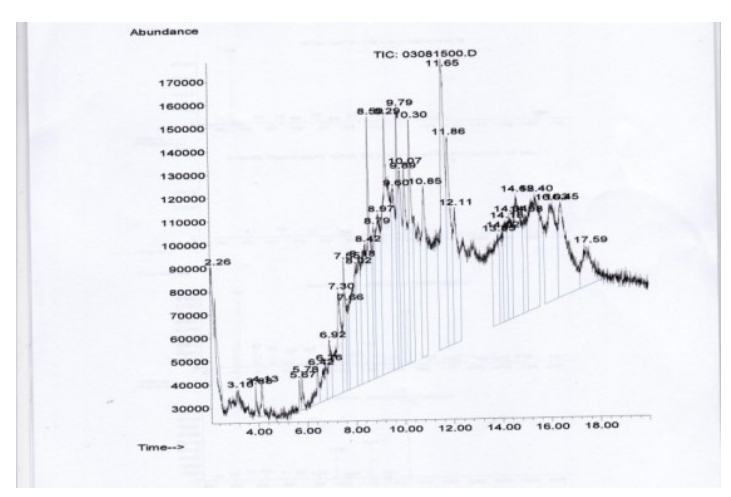

Figure 6. Chromatogram profile of acetic ethyl extract from Dicranella coarctata 


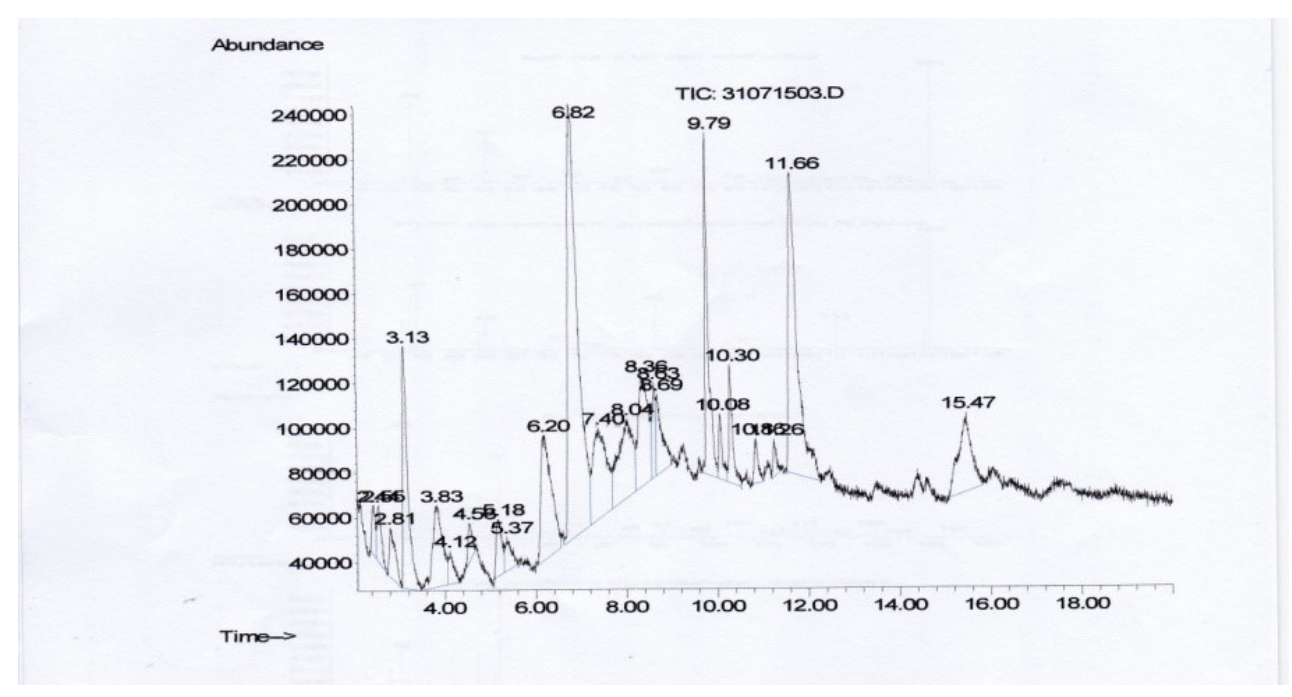

Figure 7. Chromatogram profile of methanol extract from Dicranella coarctata

Table 2. Chemical compounds from n-hexane extract of Dicranoloma reflexum

\begin{tabular}{ccll}
\hline Peak & $\begin{array}{c}\text { Retention } \\
\text { time }\end{array}$ & \multicolumn{1}{c}{ Compound name } & $\begin{array}{c}\text { Areal range } \\
(\%)\end{array}$ \\
\hline 1 & 8.217 & Naphthali Azulene & 2.67 \\
2 & 8.354 & Naphthalene & 0.21 \\
3 & 8.383 & Naphthalene & 0.14 \\
4 & 8.423 & Naphthalene & 0.04 \\
5 & 8.440 & Naphthalene & 0.03 \\
6 & 14.311 & Pentanoic acid & 0.81 \\
7 & 14.900 & 5,7-Diamino-1,2,3,4-tetrahydro-1,1,4,4,6- & 0.36 \\
& & pentamethylnaphthalene & \\
8 & 15.764 & Heptadecane & 0.71 \\
9 & 15.856 & Cyclopentanepropanoic acid & 2.51 \\
10 & 16.147 & 2',6'-Acetoxylidide & 0.48 \\
11 & 16.268 & Trimethylphenylsilane & 0.62 \\
12 & 16.353 & Nonyphenol & 1.21 \\
13 & 16.411 & 4- Nonylphenol & 0.89 \\
14 & 16.479 & Nonylphenol & 0.47 \\
15 & 16.497 & Nonylphenol & 0.68 \\
16 & 16.714 & Nonylphenol & 2.05 \\
17 & 16.851 & Phenol & 1.33 \\
18 & 16.971 & 1-Octadecene & 9.95 \\
19 & 17.143 & Eicosane & 1.66 \\
20 & 17.635 & Hexahydrofarnesyl acetone & 2.08 \\
21 & 18.202 & Nonadecane & 0.49 \\
22 & 18.499 & 1- Pentadecane & 0.26 \\
23 & 18.551 & Pentadecanoic acid & 4.17 \\
24 & 18.608 & Hexadecanoic acid & 3.70 \\
25 & 18.774 & Hexadecanoic acid & 1.18 \\
\hline
\end{tabular}


Continued of Table 2

\begin{tabular}{|c|c|c|c|}
\hline Peak & $\begin{array}{l}\text { Retention } \\
\text { time }\end{array}$ & Compound name & $\begin{array}{c}\text { Areal } \\
\text { range }(\%)\end{array}$ \\
\hline 26 & 19.129 & Dodecynilsuccinic anhydride 9 Nonadecene & 0.38 \\
\hline 27 & 19.220 & 1- Octadecene & 12.53 \\
\hline 28 & 19.541 & I-Propyl 14 methyl-Pentadecanoate & 0.15 \\
\hline 29 & 20.376 & Oleic acid & 1.15 \\
\hline 30 & 20.582 & Octadecanoic acid & 2.10 \\
\hline 31 & 21.160 & 1- Octadecene & 8.25 \\
\hline 32 & 22.402 & Eicisanoic acid & 0.39 \\
\hline 33 & 22.699 & 9-Tricosene $(Z)-$ & 0.31 \\
\hline 34 & 22.905 & 9-Tricosene $(Z)-$ & 3.89 \\
\hline 35 & 23.775 & 9-Tricosene $(Z)-$ & 0.22 \\
\hline 36 & 24.015 & 9-Octadecanoic acid & 0.30 \\
\hline 37 & 24.204 & $\begin{array}{l}\text { 2-Pyrazoline, 5-hydroxy-3-metyl-5-trifluromethyl1-2- } \\
\text { (2-isopropyl-5-methylphenox }\end{array}$ & 0.32 \\
\hline 38 & 24.490 & 1-nonadecene & 1.28 \\
\hline 39 & 24.656 & Elaidic acid & 0.07 \\
\hline 40 & 24.776 & 9-Tricosene $(Z)-$ & 0.02 \\
\hline 41 & 24.908 & Elaidic acid & 0.13 \\
\hline 42 & 25.051 & 9-Octadecenoic acid & 0.14 \\
\hline 43 & 25.160 & 9-Octadecenoic acid & 0.07 \\
\hline 44 & 25.360 & Elaidic acid & 0.35 \\
\hline 45 & 25.526 & 9-Octadecenoic acid & 0.45 \\
\hline 46 & 25.972 & Elaidic acid & 2.27 \\
\hline 47 & 26.750 & 9-Octadecenoic acid & 10.93 \\
\hline 48 & 26.842 & 9-Octadecenoic acid & 0.68 \\
\hline 49 & 26.996 & 9-Octadecenoic acid & 3.85 \\
\hline 50 & 27.151 & 2,3-Dihydroxypropyl elaidate & 1.02 \\
\hline 51 & 27.340 & 9-Octadecenoic acid & 2.96 \\
\hline 52 & 27.534 & Elaidic acid & 2.09 \\
\hline 53 & 27.574 & Oleic acid & 0.54 \\
\hline 54 & 27.620 & n-Propyl 9- Octadecanoate & 0.41 \\
\hline 55 & 27.689 & 16-Octadecenoic acid & 0.61 \\
\hline 56 & 27.717 & n-Propyl 9- Octadecanoate & 0.50 \\
\hline 57 & 27.803 & 9-Octadecenoic acid & 0.83 \\
\hline 58 & 27.843 & 9-Octadecenoic acid & 0.26 \\
\hline 59 & 27.889 & 9-tricosane, $(Z)-$ & 0.34 \\
\hline 60 & 28.084 & 9-Octadecenoic acid & 0.98 \\
\hline 61 & 28.358 & i-Propyl 9-Octadecenoate & 0.55 \\
\hline
\end{tabular}


Table 3. Chemical compounds from acetic ethyl extract of Dicranoloma reflexum

\begin{tabular}{cclc}
\hline Peak & $\begin{array}{c}\text { Retention } \\
\text { time }\end{array}$ & \multicolumn{1}{c}{ Compound name } & $\begin{array}{c}\text { Areal } \\
\text { range (\%) }\end{array}$ \\
\hline 1 & 7.730 & Cyclohexene,3,5,5-timethyl-(CAS) & 3.97 \\
2 & 13.035 & Phenol & 13.035 \\
3 & 14.408 & Pentan-1,3-Dioldiisobutyrate,2,2,4-Trimethyl & 67.78 \\
4 & 15.798 & Heptadecane & 0.64 \\
5 & 15.907 & Allyldimethyl(prop-1-ynyl)silane & 3.77 \\
6 & 17.011 & 1-Octadecene & 2.02 \\
7 & 17.074 & 1-Octadecene & 1.00 \\
8 & 17.675 & 2-Pentadecanone,6,10,14-trimethyl- & 1.68 \\
9 & 18.242 & Nonadecane & 0.92 \\
10 & 18.545 & 4-(91Z)-N-hydroxythanimidoyl)2-methylpyridazin- & 0.70 \\
& & 3(2H)-one & \\
11 & 19.100 & 1,2-Benzenedicarboxylic acid, dibutyl ester & 1.77 \\
12 & 19.260 & 1-Octadecene & 3.81 \\
13 & 21.194 & 9-Tricosane & 1.29 \\
14 & 27.483 & 11-Octadecenoic acid & 7.81 \\
15 & 28.020 & n-Propyl 9-Octadcenoate & 1.41 \\
16 & 28.267 & Erucic acid & 0.29 \\
\hline
\end{tabular}

Tabel 4. Chemical compounds from methanol extract of Dicranoloma reflexum

\begin{tabular}{ccll}
\hline Peak & $\begin{array}{c}\text { Retention } \\
\text { time }\end{array}$ & \multicolumn{1}{c}{ Compound name } & $\begin{array}{c}\text { Areal range } \\
(\%)\end{array}$ \\
\hline 1 & 5.899 & Cycloheptane & 0.50 \\
2 & 8.411 & 4-Fluorothiophenol & 0.06 \\
3 & 8.594 & Azulene & 0.14 \\
4 & 8.680 & Naphthalene & 0.07 \\
5 & 8.835 & Naphthalene & 0.12 \\
6 & 10.632 & 1,3-Cyclopentanedione,4-hydroxy-2methyl & 0.32 \\
7 & 11.284 & Hexenoic acid & 0.15 \\
8 & 11.770 & 3-Chloropropionic acid & 0.14 \\
9 & 12.703 & 2-Furanmethanol & 0.45 \\
10 & 12.869 & 2,5-Difluorobenzenoic acid & 0.71 \\
11 & 13.023 & Heptadecanoic acid & 0.21 \\
12 & 13.224 & Phenol & 0.66 \\
13 & 13.733 & 2(4H)-Benzofuranone,5,6,7,71-tetrahydro-4,4,7a- & 0.30 \\
& & trimethyl & \\
14 & 14.236 & 2-Tetradecene & 0.94 \\
15 & 14.351 & Propanoic acid & 2.60 \\
16 & 14.946 & 8-(N-(Etfyl) amino)-5,6-dimethoxyquinoline & 0.52 \\
17 & 15.552 & Cyclotetradecane & 0.58 \\
18 & 15.781 & Cyclopentadecane & 0.45 \\
19 & 15.896 & Pentanoic acid & 2.01 \\
20 & 16.096 & n-Tetradecane & 0.46 \\
21 & 16.193 & 1-Tetradecane & 0.22 \\
22 & 16.376 & 2,6-Diisopropylnaphthalene & 1.44 \\
23 & 16.720 & 1-Octadecene & 0.17 \\
\hline
\end{tabular}


Continued of Table 4

\begin{tabular}{cclc}
\hline Peak & $\begin{array}{c}\text { Retention } \\
\text { time }\end{array}$ & Compound name & $\begin{array}{c}\text { Areal range } \\
(\%)\end{array}$ \\
\hline 24 & 17.000 & 1-Octadecene & 6.32 \\
25 & 17.561 & Cyclohexanone & 0.26 \\
26 & 17.670 & 2-pentadecanone & 2.99 \\
27 & 17.830 & 4-((1Z)-(N-Hydroxyethanimydoyl)-2- & 0.31 \\
& & methylpyridazine-3-(2H)-one & \\
28 & 18.047 & 9-Octadecenoic acid & 0.21 \\
29 & 18.230 & 2-Hexyldecanol & 0.23 \\
30 & 18.476 & 11-Octadecenoic acid & 0.32 \\
31 & 18.579 & Hexadecenoic acid & 5.27 \\
32 & 18.814 & Methyl-3-(3,5-Diterbutyl-4- & 2.75 \\
& & Hydroxyphenyl)Propionate & \\
33 & 18.997 & 1-Octadecene & 0.51 \\
34 & 18.249 & 1-Octadecene & 4.26 \\
35 & 18.798 & 9-Octadecanal & 0.21 \\
36 & 20.16 & 9-Octadecenoic acid & 0.26 \\
37 & 20.262 & Tert-Hexadenanethiol & 0.40 \\
38 & 20.405 & 9-Octadecenoic acid & 2.24 \\
39 & 20.599 & 10-Octadecenoic acid & 1.07 \\
40 & 20.977 & 9-Octadecenoic acid & 1.10 \\
41 & 21.194 & 1-Heptacosanol & 1.29 \\
42 & 22.430 & Thiosulfuric acid & 0.24 \\
43 & 22.739 & 9-Octadecenoic acid & 0.23 \\
44 & 22.934 & 9-Octadecenoic acid & 0.33 \\
45 & 26.762 & 9-Octadecenoic acid & 11.06 \\
46 & 27.586 & 9-Octadecenoic acid & 15.03 \\
47 & 27.935 & 9-Octadecenoic acid & 5.71 \\
48 & 28.576 & Elaidic acid & 10.92 \\
49 & 28.679 & Elaidic acid & 1.15 \\
50 & 29.022 & 9-Tricosane, (Z)- & 3.83 \\
51 & 29.176 & Elaidic acid & 1.95 \\
52 & 29.400 & 9-Octadecenoic acid & 3.19 \\
53 & 29.680 & Elaidic acid & 1.35 \\
54 & 29.926 & Elaidic acid & 0.64 \\
55 & 30.029 & Elaidic acid & 0.24 \\
56 & 30.098 & 9-Octadecenoic acid & 0.29 \\
57 & 30.218 & 9-Tricosane, (Z)- & 0.27 \\
58 & 30.338 & 9-Octadecenoic acid & 0.49 \\
\hline & & &
\end{tabular}


Table 5. Chemical compounds from n-hexane extract of Dicranella coarctata

\begin{tabular}{cclc}
\hline Peak & $\begin{array}{c}\text { Retention } \\
\text { time }\end{array}$ & \multicolumn{1}{c}{ Compound name } & $\begin{array}{c}\text { Areal range } \\
(\%)\end{array}$ \\
\hline 1 & 8.12 & Thiosulfuric acid & 42.52 \\
2 & 8.15 & Tetrahydroxycyclopentadienone & 2.72 \\
3 & 8.26 & 9-Octadecenoic acid & 12.83 \\
4 & 9.79 & Cyclopentane & 18.43 \\
5 & 10.85 & 9-Octadecenoic acid & 23.50 \\
\hline
\end{tabular}

Table 6. Chemical compounds from acetic ethyl extract of Dicranella coarctata

\begin{tabular}{ccll}
\hline Peak & $\begin{array}{c}\text { Retention } \\
\text { time }\end{array}$ & \multicolumn{1}{c}{ Compound name } & $\begin{array}{c}\text { Areal range } \\
(\%)\end{array}$ \\
\hline 1 & 2.26 & Cyclotetrasiloxane & 0.74 \\
2 & 3.10 & 2-hexanamine, 4 methyl & 0.18 \\
3 & 3.88 & 2-hexanamine, 4 methyl & 0.18 \\
4 & 4.13 & Naphthalene & 0.32 \\
5 & 5.67 & Benzeneethanamine & 0.21 \\
6 & 5.78 & Benzeneethanamine & 0.23 \\
7 & 6.42 & Benzeneethanamine & 0.57 \\
8 & 6.76 & 2,4(1H,3G=H)-Pyrimidinedione & 0.80 \\
9 & 6.92 & Metraraminol bitartrate & 0.92 \\
10 & 7.30 & o-hydroxybiphenyl & 2.74 \\
11 & 7.55 & (cis)-2-nonadecene & 1.60 \\
12 & 7.65 & Tetrahydroxycyclopentadienone & 0.91 \\
13 & 8.02 & 2Hydroxymino-N-(P-methoxyphen) & 3.25 \\
14 & 8.18 & 2Hydroxymino-N-(P-methoxyphen) & 2.33 \\
15 & 8.42 & Hexadecanoic acid & 3.58 \\
16 & 8.59 & Cyclophenyl 2-Methylenebutanyl & 3.94 \\
17 & 8.80 & 2Hydroxymino-N-(P-methoxyphen) & 1.63 \\
18 & 8.97 & Hydroxymethapyrilene & 4.03 \\
19 & 9.29 & E-15-Heptadecenal & 8.61 \\
20 & 9.61 & 1-Hentetracontanol & 3.28 \\
21 & 9.79 & 1,2-Cyclohexadediol & 2.61 \\
22 & 9.90 & 14-BETA-H-Pregna & 2.89 \\
23 & 10.07 & Trans pinene & 3.62 \\
24 & 10.30 & Citronellyl valerat & 3.56 \\
25 & 10.85 & Cyclohexane & 3.42 \\
26 & 11.65 & Hexadecanoic acid & 7.21 \\
27 & 11.86 & 1-Pentadecene & 3.85 \\
28 & 12.11 & (trans)-2-nonadecene & 3.47 \\
29 & 13.85 & 17-Pentatriacontene & 2.37 \\
30 & 14.02 & 4-Hexenoic acid & 1.45 \\
31 & 14.19 & 4-Hexenoic acid & 1.80 \\
32 & 14.34 & 17-Pentatriacontene & 2.10 \\
33 & 14.62 & 17-Pentatriacontene & 4.46 \\
34 & 14.97 & 17-Pentatriacontene & 1.86 \\
35 & 15.40 & 9-Octadecenal & 5.15 \\
36 & 16.03 & 1-Octadecene & 5.05 \\
37 & 16.45 & 1-Eiocosene & 4.58 \\
38 & 17.59 & Dimer of Coleon F & 1.94 \\
\hline & & &
\end{tabular}


Table 7. Chemical compounds from methanol extract of Dicranella coarctata

\begin{tabular}{cclc}
\hline Peak & $\begin{array}{c}\text { Retention } \\
\text { time }\end{array}$ & \multicolumn{1}{c}{ Compound name } & $\begin{array}{c}\text { Areal range } \\
(\%)\end{array}$ \\
\hline 1 & 2.44 & 1,2,3,4-Tetrahydroxybutane & 0.88 \\
2 & 2.56 & Pentanal & 1.24 \\
3 & 2.81 & N,N'-Dimethylpiperazine & 1.51 \\
4 & 3.13 & Cyclopentanone & 6.34 \\
5 & 3.84 & Propylamine & 4.28 \\
6 & 4.13 & Methyl 2-(phenylsulfonyl)-5-deu & 0.90 \\
7 & 4.55 & 2-Furancarboxaldehyde,5-(hydro & 0.78 \\
8 & 5.18 & N-(2-methoxycarbonylethylidene) & 1.61 \\
9 & 5.37 & (E)-4-chloro-2,3-dimethyl & 1.04 \\
10 & 6.20 & Isothiazole & 6.93 \\
11 & 6.82 & 6,6 Dideutero-nonen-1-ol & 22.92 \\
12 & 7.40 & Nonanoic acid & 6.47 \\
13 & 8.05 & Benzene,1-methyl-2-(2-propenyl & 5.85 \\
14 & 8.36 & 1H-indene,2,3-dihydro- & 5.83 \\
15 & 8.63 & Undecanal & 1.57 \\
16 & 8.69 & Hexadecanoic acid & 2.42 \\
17 & 9.80 & Neophytadiene & 5.73 \\
18 & 10.08 & 1-formyl-2,2,6-trimethyl-3,(3-m & 1.16 \\
19 & 10.30 & 1,8-Nonadiene,2,8-dimethyl- & 2.23 \\
20 & 10.86 & 14-Beta-H-Pregna & 0.89 \\
21 & 11.26 & Methyl-3-(3,5-Diterbutyl-4-Hyd & 0.61 \\
22 & 11.66 & n-Hexadecanoic acid & 13.62 \\
23 & 15.48 & Cyclopropaneoctanal & 5.19 \\
\hline
\end{tabular}

Based on GC-MS analysis, n-hexane extract of Dicranoloma reflexum was found to consist 61 peaks, indicating that it contained 61 compounds. Main compound found was 1-octadecene with areal total $12.53 \%$ (Table 2). Acetic ethyl extract of Dicranoloma reflexum contained 16 compounds, the main compound was phenol with areal range $13.035 \%$ (Table 3). Methanol extract of Dicranoloma reflexum contained 58 compounds. Main compound found was 9-octadecanoic acid with areal range of $15.03 \%$ (Table 4 ).

1-octadecene was a hydrocarbon compound usually found from Moringa oleifera. This compound possessed antioxidant, anti-bacterial, and anti-fungal properties towards Bacillus cereus, Escherichia coli, Pseudomonas aeruginosa, Penicillium aurantio, Penicillium griceum, Penicillium expansum, Penicillium digitatum, and Aspergillus niger (Maruffo et al., 2013). N-hexane extract of Prunus dome was also found to contain 1-octade 91 This compound had anti-bacterial activity towards Salmonella and anti-fungal towards Microsporum canis. In addition, this compound also had potential as antioxidant. Other compound found was phenol with chemical formula of $\mathrm{C}_{6} \mathrm{H}_{5} \mathrm{OH}$ and possessed hydroxyl group bound to phenyl ring on its structure. Cowan (1999) reported that phenol compound without hydroxyl group had higher antibacterial activity caused of higher affinity towards microbes plasma membrane. Qoumarin and quercetin were phenolic compounds with anti-bacterial activity 
towards Escherichia coli, Enterobacter aerogenes, Salmonella typhimurium, and Salmonella infantis (Nitiema et al., 2012).

Another compound identified was 9octadecanoic acid with chemical formula $\mathrm{C}_{19} \mathrm{H}_{3} 6 \mathrm{O}_{2}$ and possessed anti-oxidative and anti-carcinogenic biological activities (Syeda et al., 2011 and Hema et al., 2011). N-hexadecanoic acid had chemical formula of $\mathrm{C}_{16} \mathrm{H}_{32}$ and biological properties as anti-fungi, anti-oxidant, hypocholesterolemic, nematicide, and anti-androgenic flavor, haemolitic-5-alpha reductase inhibitor, anti-microbe, and anti-malaria (Hema et al., 2011 and Pietro et al., 2010). The ethanol extract of leaves of Indigofera suffruticosa containing nhexadecanoic acid (9.83\%) and 9octadecanoic acid (10.21\%) (Vijisaral and Arumugam, 2014). The same compound is also found in the ethanol extract of beans, respectively $7.02 \%$ and $0.89 \%$. Markkas and Govindharajala (2015) showed that the methanol extract of Mollugo cerviana containing hexadecanoic acid and 9-octadecanoic

\section{References}

Aja PM. Nwachukwu N. Ibiam US, Igwenyi IO. Offor LE. and Orji UO. 2014. Chemical Constituents of Moringa oleifera Leaves and Seeds from Abakaliki, Nigeria. American Journal of Phytomedicine and Clinical Therapeutics. 2(3):310-321.

Alam A, Srama V, Rawat KK, and Verma PK. 2015. Bryophytes-the Ignored Medicinal plants. Sikkim Manipal University. Vol2. No.1:299-315.

Asakawa Y. 2007. Biologically Active Compounds from Bryophytes. Faculty of Pharmaceutical Sciences. Tokushima Bunri University. Yamashiro-cho. Tokushima. Japan.

Asakawa Y. 2008. Liverworts-Potential Source of Medicinal Compounds. Current Pharmaceutical Design 14(29): 3067-3085. acid. Harkati et al. (2012) reported that Scorzonera undulata containing hexadecanoic acid (42.2\%) and 9octadecanoic acid (7.7\%). Aja et al. (2014) reported that the methanol extract of Moringa oleifera contains 9octadecanoic acid is more higher $(20.8 \%)$ than hexadecanoic acid (1.31\%)

Another one compound found was thiosulphuric acid with chemical formula of $\mathrm{C}_{20} \mathrm{H}_{7} \mathrm{NO}_{3} \mathrm{~S}_{2}$ and 157 molecular weigh. This compound was also identified from methanol extract of Clerodendron phlomidis ((Lakshmi dan Viji Stella Bai, 2015). Kumar et al (2011) reported that methanol and acetone extract of Spirulina platensis also contained E-15 Heptadecanal. This compound possessed anti-bacterial properties against Staphylococcus aureus and Salmonella typhimurium.

\section{Acknowledgement}

Author would like to thank 2015 DIPA DITLITABMAS of Airlangga University for funding this study.

Cowan MM. 1999. Plant Products as Antimicrobial Agents. Clinical Microbiology Reviews 12(4): 564570.

Harkati B, Akkae S, Franca MGD. 2012. Composition of The Volatile Components Extracted from The Roots of Scorzonera undulata ssp deliciosa (Guiss) Maire: from Algeria. Green and Sustainable Chemistry (2): 59-61.

Hema R, kusmaravel s, and Alagusundaram. 2011. GC/MS Determination of Bioactive components of Murraya koenigii. Journal of American Science. (7)1. 
Kumar V, Bhatnagar AK, and Srivastava JN. 2011. Antibacterial Activity of Crude Extracts of Spirulina platensis and its structural Elucidation of Bioactive compound. Journal of Medicinal Plants research Vol 5(32). Pp. 7043-7048

Lakshmi V and Viji Stella Bai G. 2015. Determination of Biologically Active Compounds in Clerodendrum phlomidis (L.) leaf Extract Using GC-MS. International journal of multidisciplinary research and Development: 2(1):294-300

Mahmood A, Ahmed R, and kosar s. 2009. Phytochemical Screening and Biological Activities of the Oil Components of Prunus domestica Linn. Journal of saudy Chemical Society Vol 13. Issue 3: 273-277.

Marimuthu K, Nagaraj N, Ravi O. 2014. GC-MS Analysis of Phytochemicals, Fatty Acids and Antimicrobial Potency of Dry Christmas Lima Beans. Int. J. Pharm. Sci. Rev.Res 27(2):63-66.

Markkas N and Govindharajalu M. 2015. Determination of Phytocomponents in The Methanolic Extract of Mollugo cerviana by GC-MS Analysis. International Journal of Research in Biological sciences. 5(4): 26-29.
Maruffo T, Nazzaro F, Mancini E, Fratianni F, coppola F, De martino $\mathrm{L}$, Agostinho AB, and de feo V. 2013. Chemical Composition and Biological Activity of The essential oil from Leaves of Moringa oleifera Lam. Cultivated in Mozambique. Molequles 18: 10989-11000.

Pietro Z, Maurizio S, Maurizio B. Antonella M, sergio R, carmen $\mathrm{f}$, and Felice S. 2010. Essential Oil composition of stems and Fruits of Carraluma Europea N.E.Br. (Apocynaceae). Molecules.15:627638.

Saxena DK and Harinder. 2004. Uses of Bryophytes. Resonance. pp. 56-65.

Syeda FA, Habib-Ur-Rehman, Choudahry MI, and Ata-ur-rahman. 2011. Gass chromatography Mass Spectra (GCMS) Analysis of Petroleum eter Extract (Oil) and Bioassys of Crude Extracts of Iris germamica. International Journal of Genetics and Molrcular Biology 3(7):95-100.

Vijisaral Edand Arumugam S. 2014. Analysis of Bioactive Constituents of Indigofera suffruticosa Leaves. Journal of Chemical and Pharmaceutical research 6(8): 294300. 